\title{
3.2 THE COMPARATIVE PROPERTIES OF THE PULSARS
}

\author{
A. G. LYNE
}

University of Manchester, Nuffield Radio Astronomy Laboratories, Jodrell Bank, U.K.

\begin{abstract}
This paper reviews the outstanding characteristics of the pulsars and, in particular, compares the properties of the Crab Nebula pulsar with those of the other pulsars. The Crab pulsar is unique in that it has a remarkably short period, emission outside the radio band, some strange amplitude variations and an abnormally large interpulse.
\end{abstract}

\section{Introduction}

This paper attempts to summarise some of the basic properties of the pulsars. In particular, I shall try to indicate in what ways the Crab pulsar fits into the morphology of the less endowed objects. I shall not discuss the polarisation characteristics of the radio pulses as Dr. Moffet will do that.

All the pulsars are seen through the interstellar medium which modifies their radiation characteristics. Some properties such as fine structure in the frequency spectrum of the radiation, certain time scales in the intensity variations and the dispersion in arrival times of the pulses are for the most part understood. The effects can usually be identified or removed from the observations so that we can deduce what the radiation would look like in the absence of the haze of the interstellar medium. One effect which cannot be removed was mentioned yesterday by Prof. Drake who showed that the Crab pulsar pulses are lengthened drastically at low frequencies. This effect is attributed to multiple path transmission in the interstellar medium; it has been studied recently at Jodrell Bank, using recently discovered pulsars with large dispersion measures. Figure 1 shows how drastic the effect can be even at quite high frequencies. I would point out that the data is quite consistent with the convolution of the natural pulse width indicated (but still slightly lengthened) at $610 \mathrm{MHz}$, with a simple exponential of the form $\mathrm{e}^{-t / \tau}$, where

$$
\tau \sim \tau_{0}\left(\frac{f_{0}}{f}\right)^{n} \text { and } n \sim 3-4 .
$$

I would point out that there is no clear cut dependence of $\tau_{0}$ on dispersion measure $(D M)$ as was suggested by Prof. Drake. The effect is not detectable in some pulsars with large dispersion measures such as JP $2003\left(\int N \mathrm{~d} l=200 \mathrm{pc} \mathrm{cm}^{-3}\right)$ but shows very clearly in JP $1946\left(\int \mathrm{N} \mathrm{d} l=130\right)$ in Figure 1. The data is nevertheless consistent with a dependence upon the square of the $D M$ as expected from some theoretical considerations, but there is a wide scatter among pulsars of similar dispersion measure.

I shall now talk about the properties of the radiation we would see in the absence of the intervening medium. 


\section{Periodicity}

The most notable property of the pulsars is the periodicity in arrival times. The periods range from $0.03 \mathrm{sec}$ for NP 0532 to $4 \mathrm{sec}$ for NP 0527. The stability of the pulsations are such that the arrival times of pulses from a number of pulsars can now be confidently predicted over periods of a year to an accuracy of a few milliseconds or better. Reichley, Downs and Morris (1970) and Hunt (1969) have provided rates of change of period for more than a dozen long period pulsars.
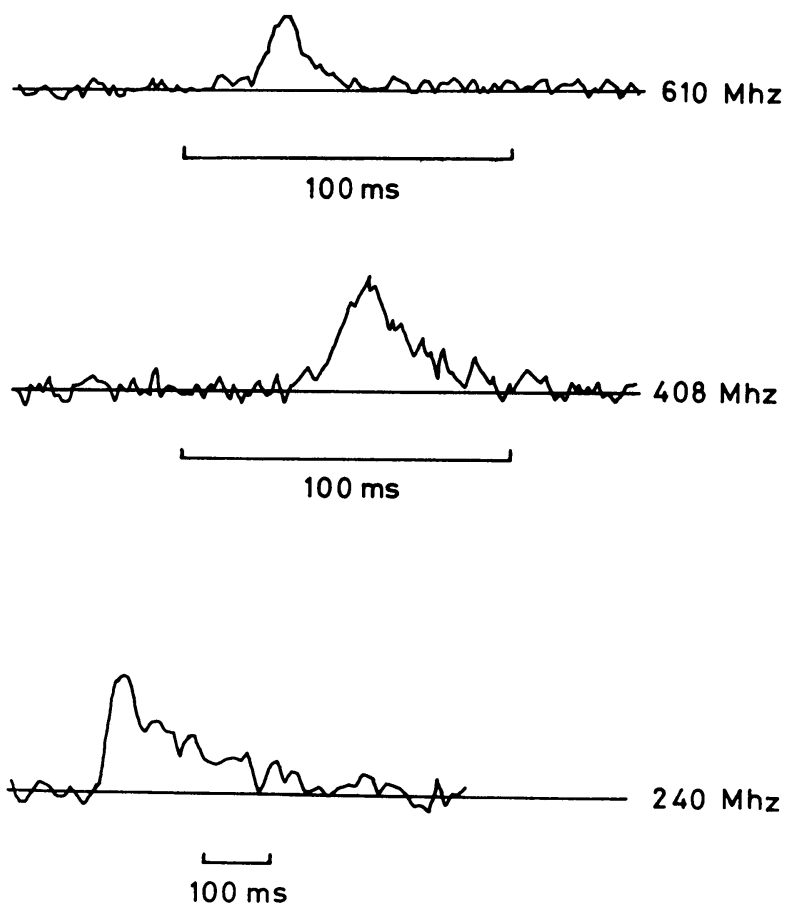

Fig. 1. Pulse broadening in JP 1946 due to multipath interstellar propogation.

Deviations from a secular slow down rate are the exception rather than the rule and the dramatic changes seen in PSR 0833-45 last year (Reichley and Downs, 1969; Radhakrishnan and Manchester, 1969) and the complicated perturbations in NP 0532 that we heard about yesterday have not been observed in other pulsars.

Figure 2 shows a plot of $\dot{P}$ against $P$ for all pulsars with known $\dot{P}$. Apart from the Crab and Vela pulsars there is no clear relationship.

Figure 3 shows a slightly clearer relationship between $\dot{P}$ and the luminosity. Again, the Crab and Vela pulsars in the top of the diagram are chiefly responsible for the correlation. It has been suggested that this is due to a dependence of both variables upon the magnetic field in the source (Hunt, 1969). 


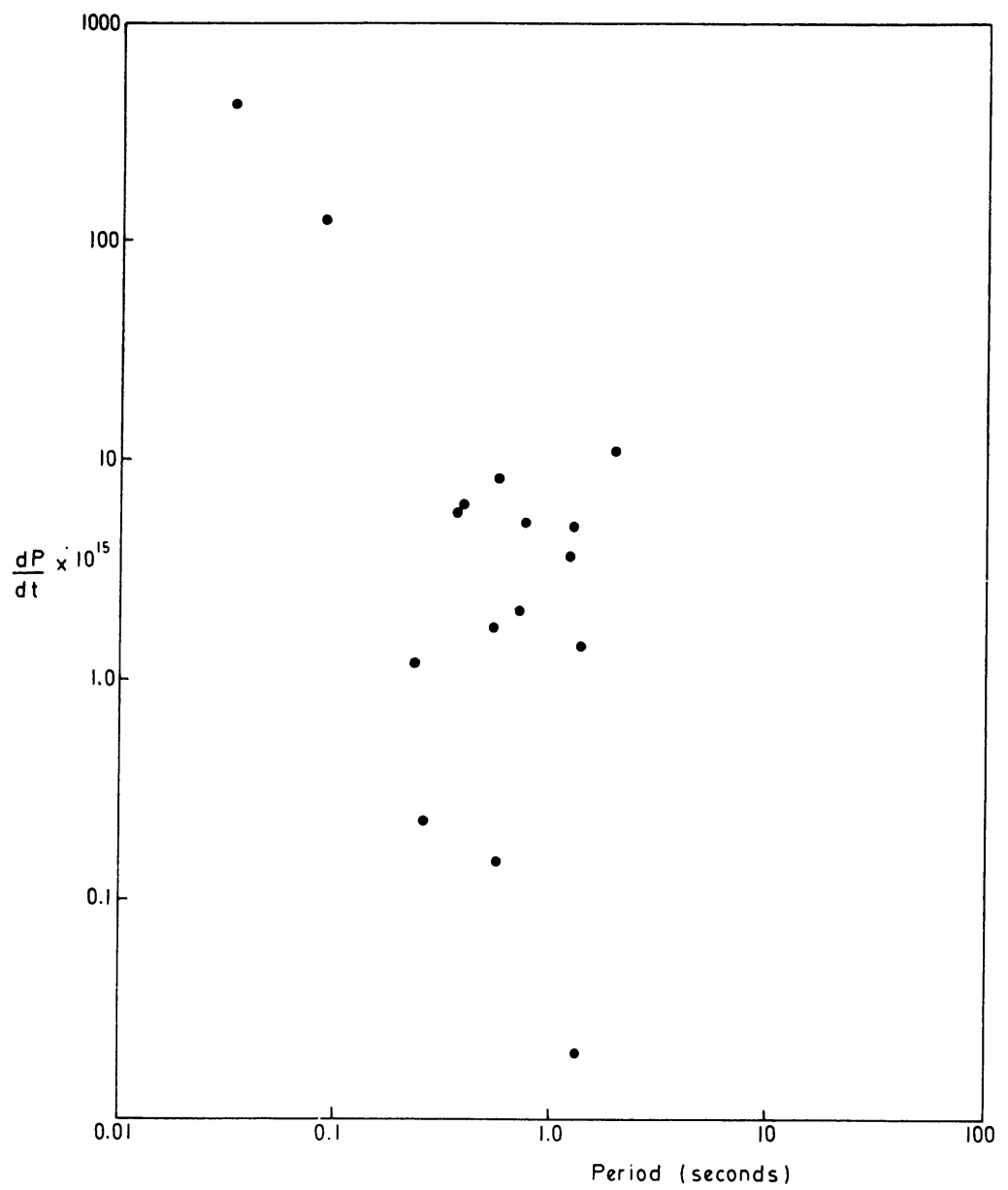

Fig. 2. Rate of change of period plotted against period for 16 pulsars.

\section{Mean Profiles}

One other well defined characteristic of a pulsar is the mean pulse profile when integrated over several hundred pulses. Such a profile is stable and is quite unique to a particular pulsar. Figure 4 shows the wide range of profiles encountered at $408 \mathrm{MHz}$.

Not immediately obvious from these profiles is a very close relationship between the pulse duration and the period. Figure 5 shows the equivalent width of the pulses, defined as the total impulsive energy per period divided by the peak impulsive power, plotted as a function of period. The straight line corresponds to pulses having a width, $w_{p}$, of $2.6 \%$ of the pulse repetition period. The most striking point about this is that all but one or two pulsars lie within a factor of two of this line.

If the pulses are interpreted as being due to the sweeping of a beam across the Earth, then the angular width of this beam is $9^{\circ}$ of longitude, with a standard deviation of 
about $3.5^{\circ}$. Any proposed emission mechanism must be able to account for this narrow range of beam-widths.

It is also clear that there is little dependence of the duty ratio upon the period. The mean duty ratio of the 14 pulsars with the shortest periods is $2.8 \%$ while that of the other 14 is $2.6 \%$. In this respect, therefore, the long period pulsars look just like

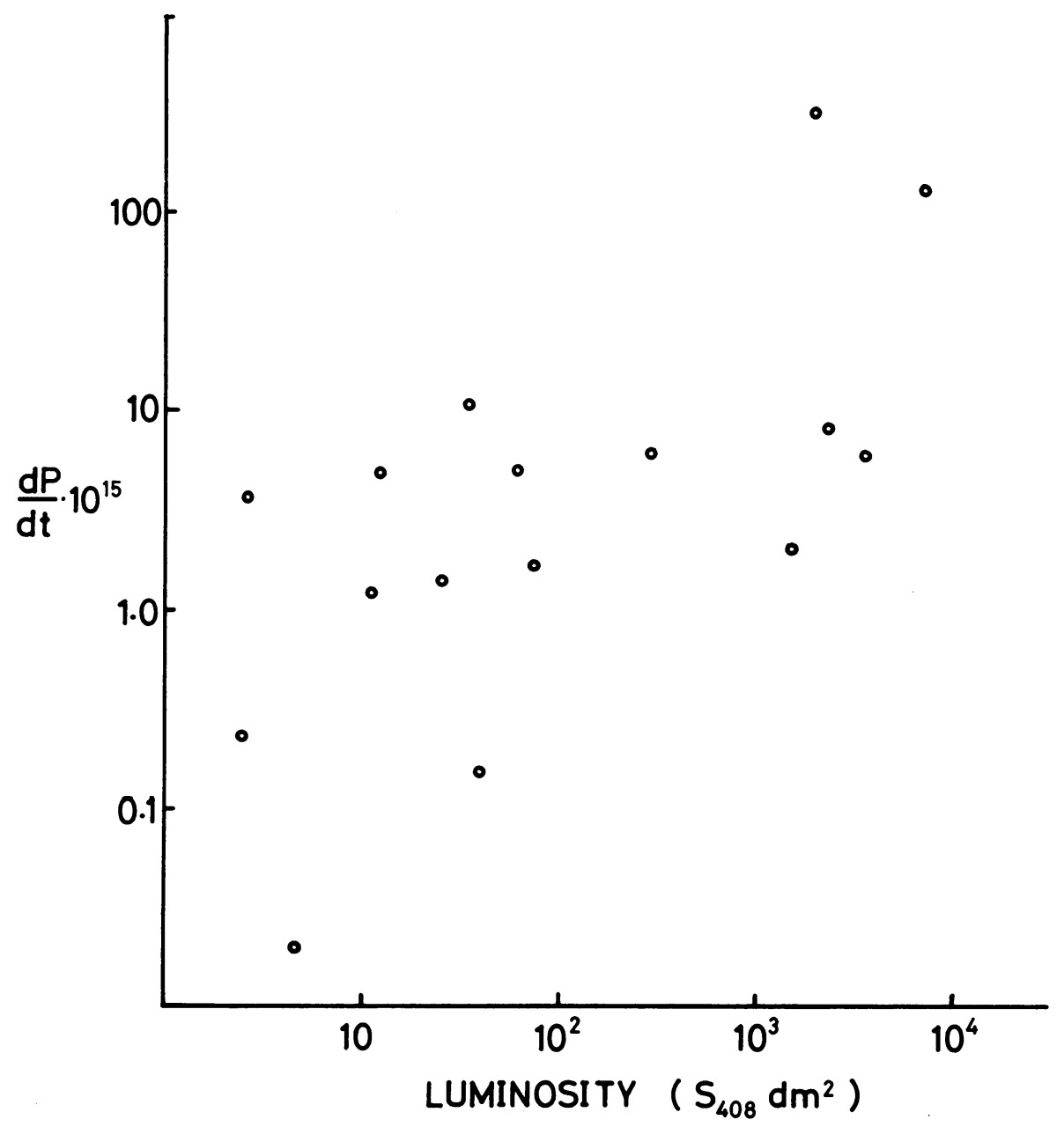

Fig. 3. Rate of change of period plotted against luminosity.

slow, short period ones. If period is any measure of age, then we can clearly say that the angular width of the beam does not evolve significantly with it.

Both the Crab and Vela pulsars have very typical duty ratios and are very similar to the others in this respect.

Figure 6 shows a weak dependence of the duty ratio upon luminosity, measured as the product of the flux at $408 \mathrm{MHz}$ and the square of the dispersion measure. The 


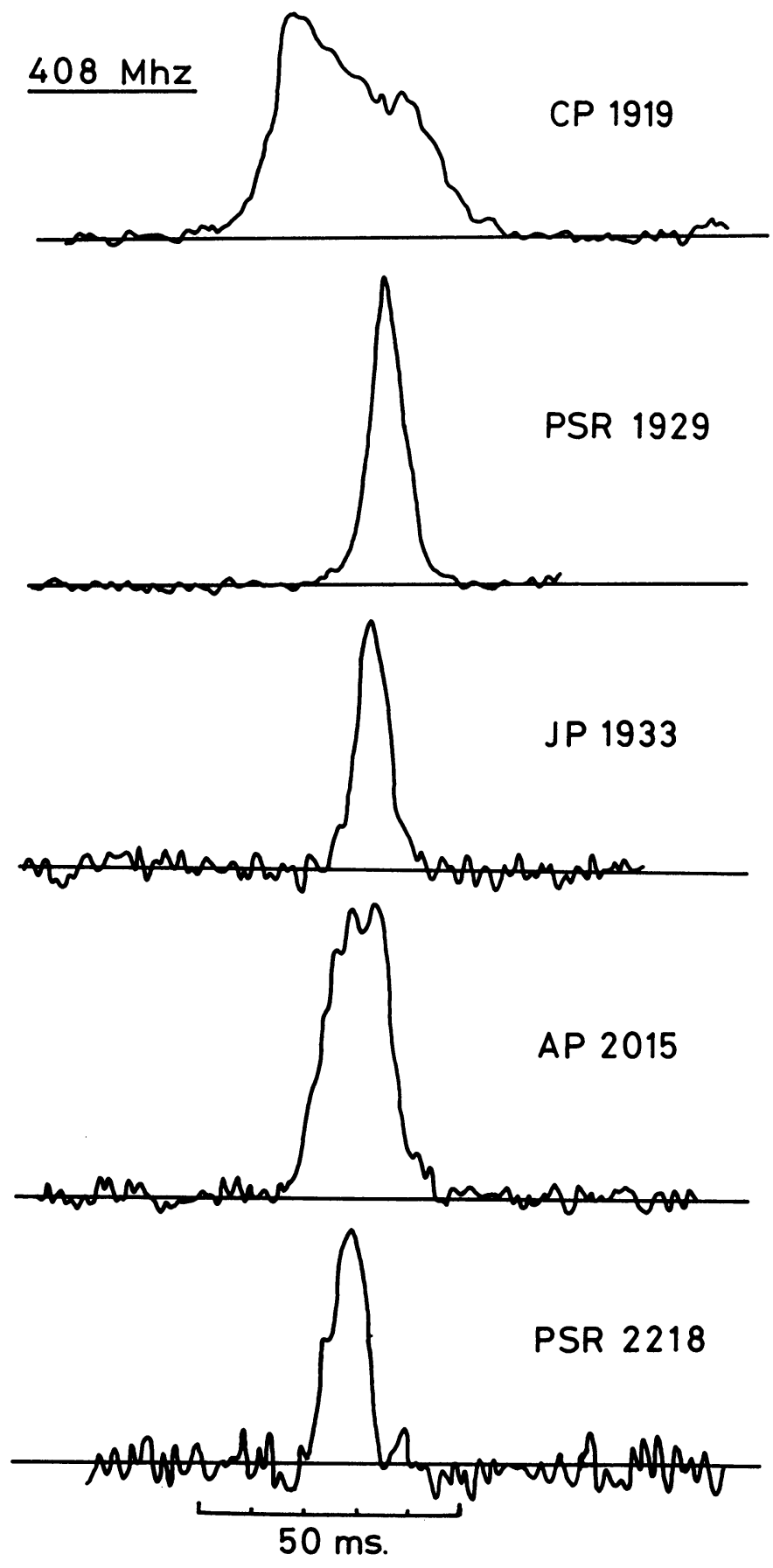

Fig. 4a. 

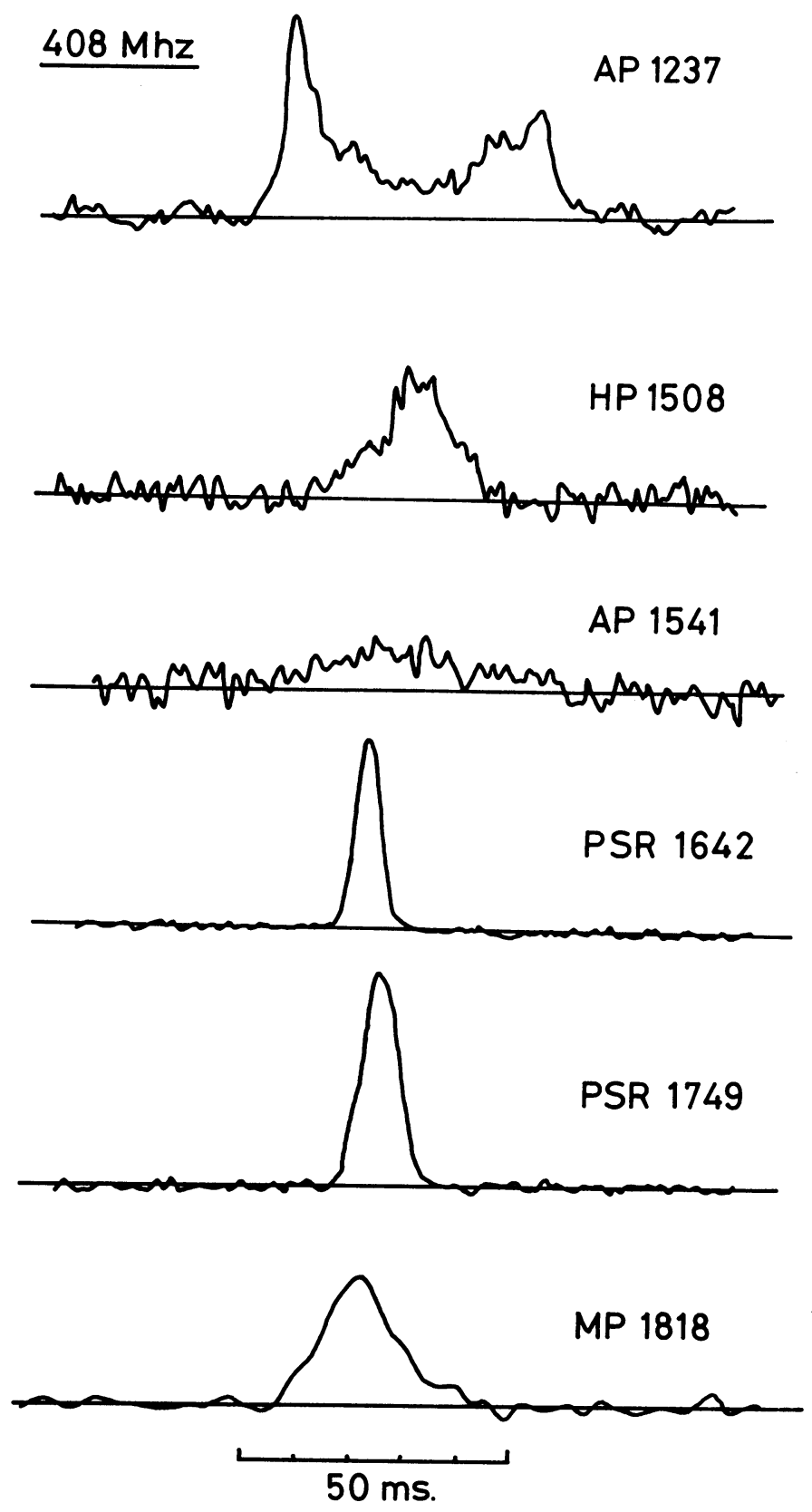

Fig. 4b. 
correlation is significant and the dependence takes the form

$$
\frac{w_{p}}{P} \propto L^{-0.1}
$$

Thus the most luminous objects tend to have slightly narrower beams. For this I offer no explanation.

I might add that there are no selection effects which can seriously affect these relationships.

The mean profiles, although well defined, often change slightly with frequency.
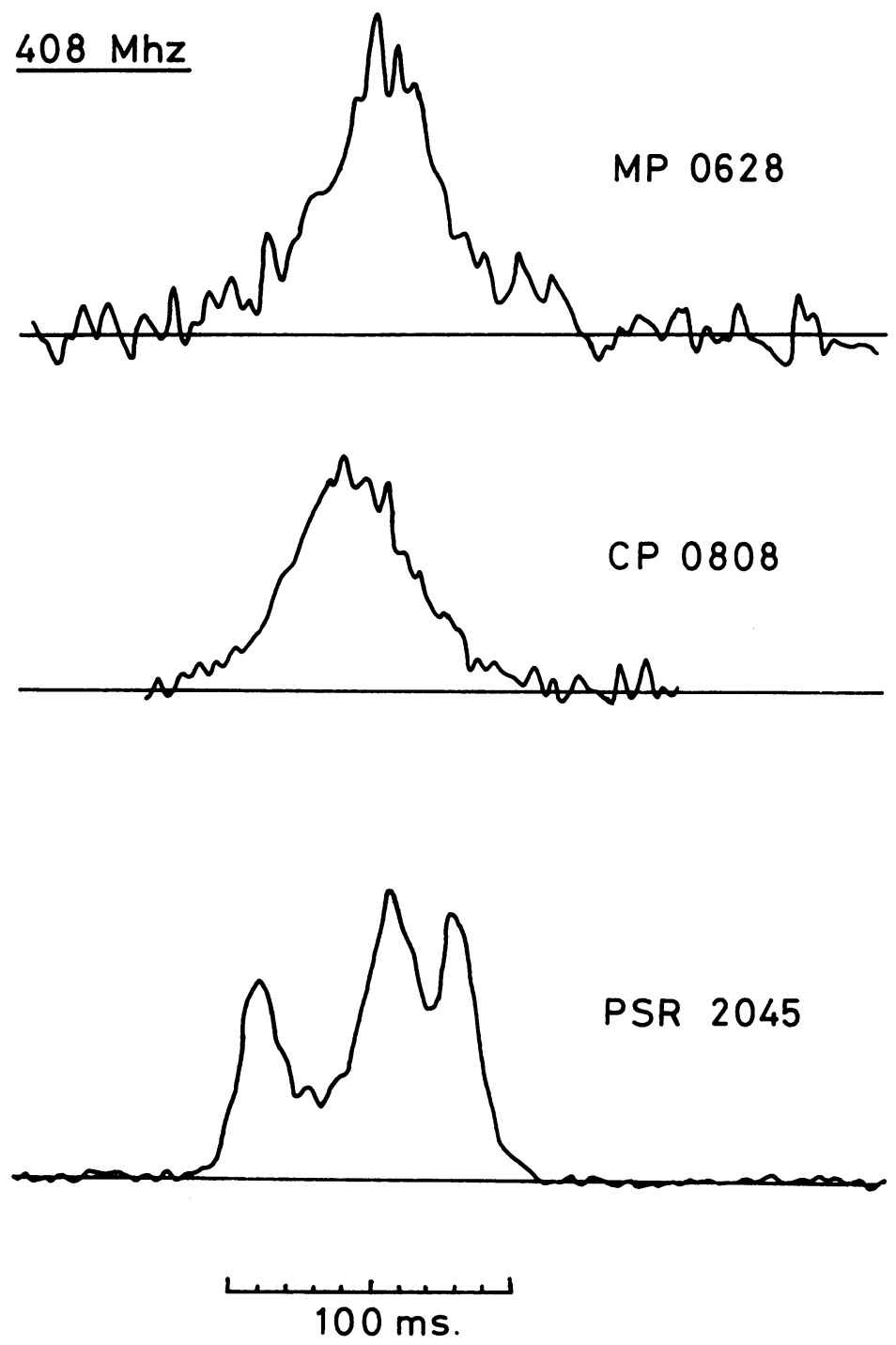

Fig. 4c.

PSR 2045 

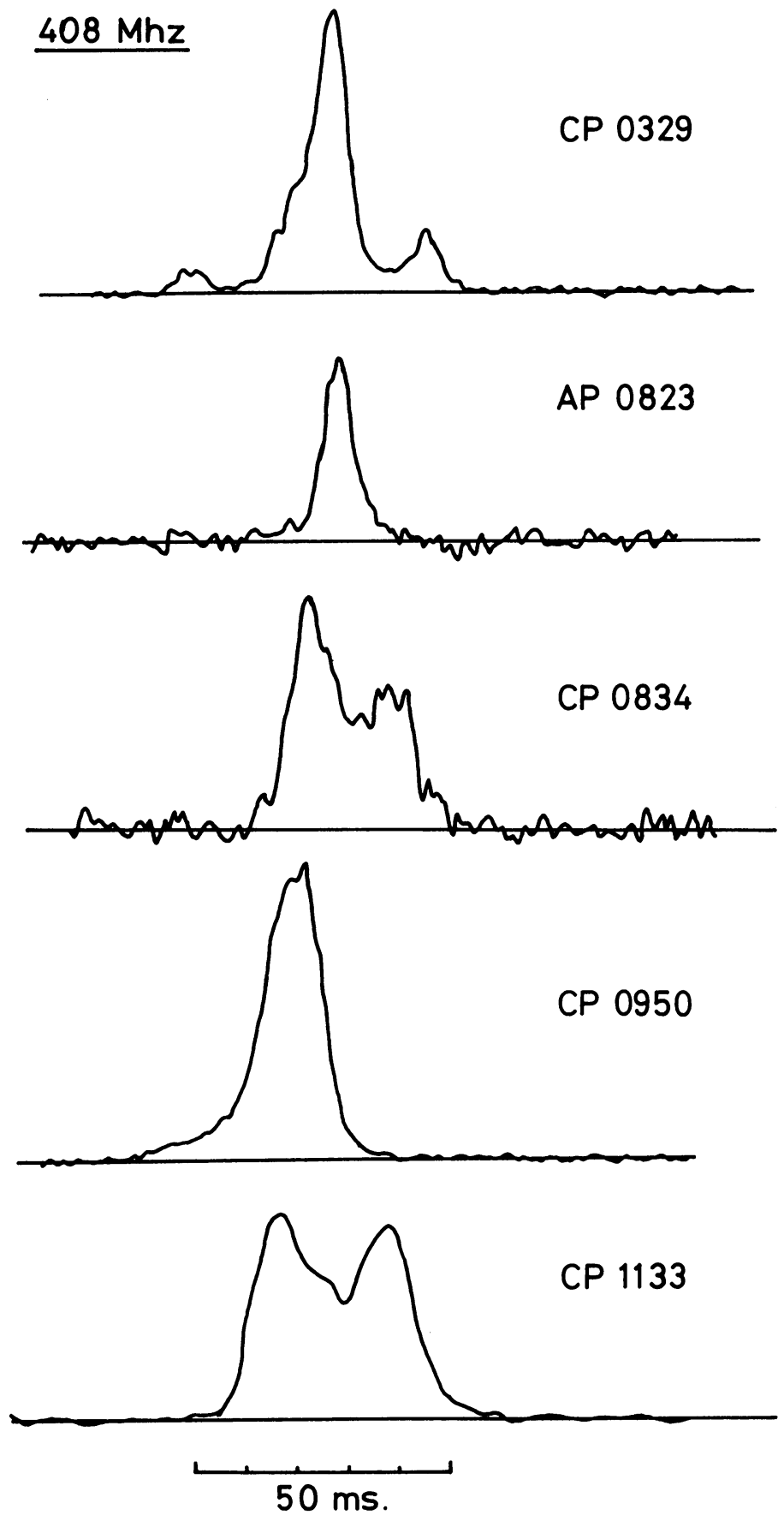

Fig. 4d.

Fig. 4a-d. Mean pulse profiles averaged over about 1000 pulses for a number of pulsars. 
The best example is shown in the Figure 7, taken from Craft and Comella (1968). Here, the separation of the two components increases as $f^{-0.25}$ and the width of the first component increases at a similar rate. The width of the second is nearly constant. Other pulsars, such as NP 0527 and PSR 2045 show similar behaviour with similar or smaller exponents. Many of the profiles in Figure 4 were obtained by Dr. B. J. Rickett.

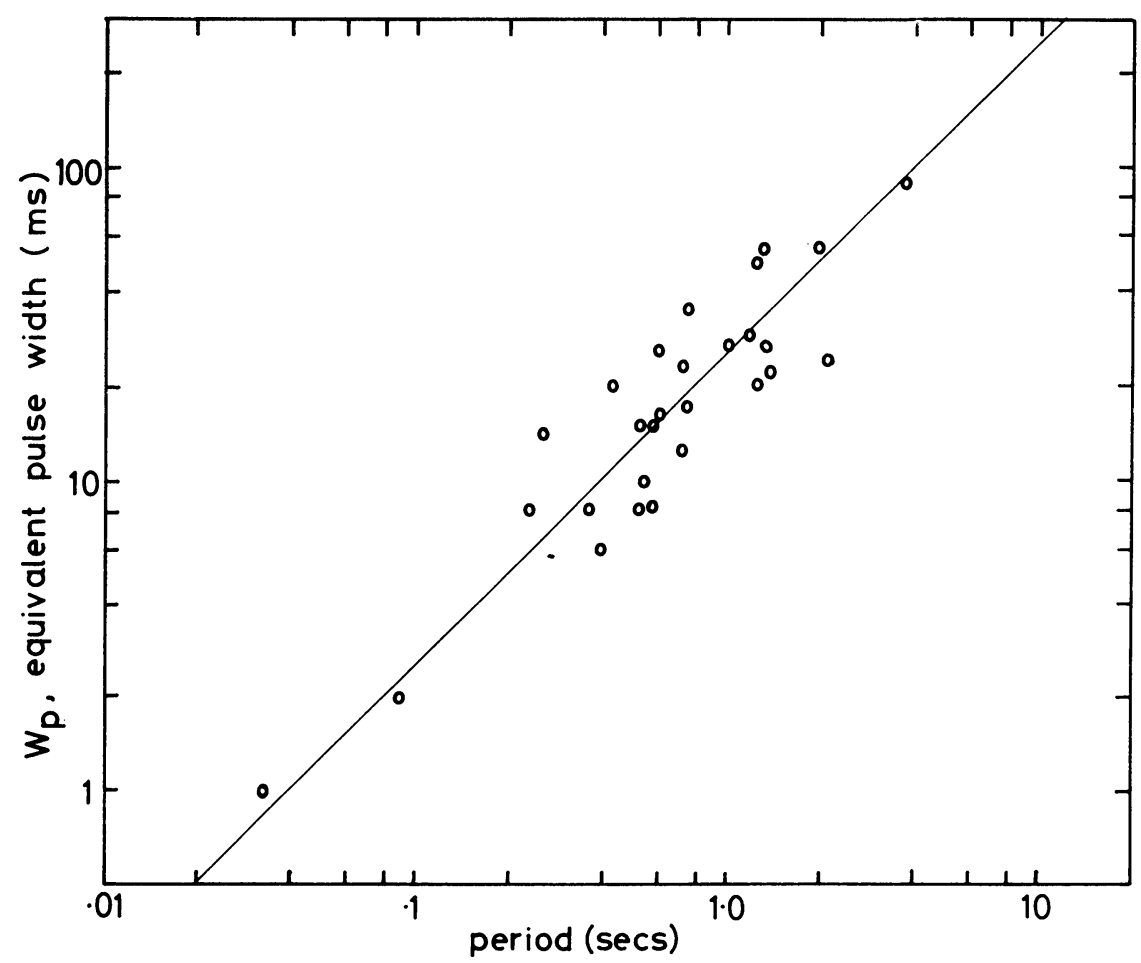

Fig. 5. Equivalent pulse width plotted against period.

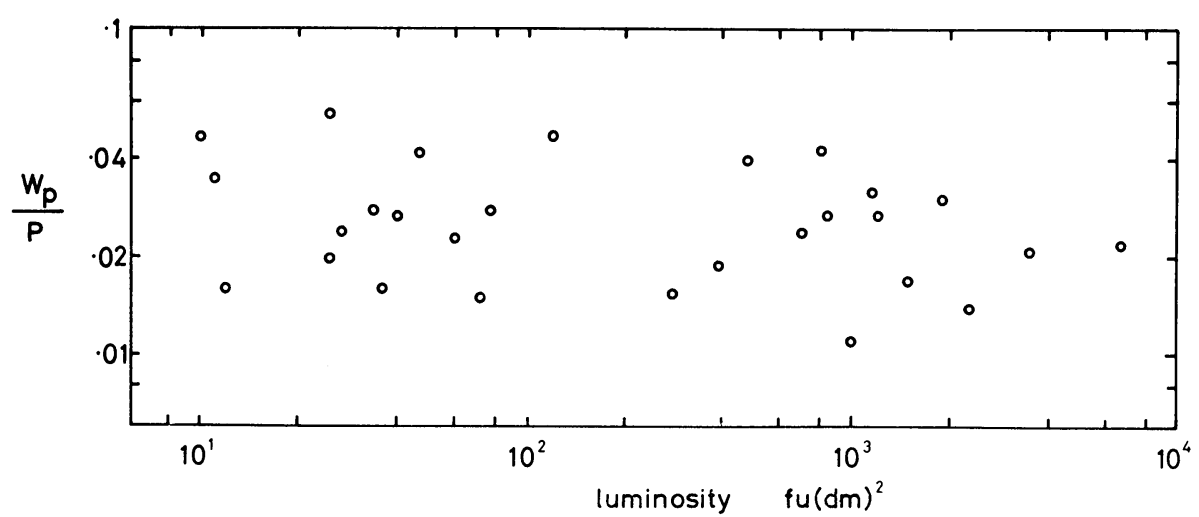

Fig. 6. The duty ratio of a number of pulsars plotted against their luminosity. 


\section{Intensity Variations}

Although mean pulse-shapes are very stable, individual pulses vary dramatically in both intensity and in shape. The nature of the intensity variations changes considerably from one pulsar to the next. Figure 8 shows the autocorrelation functions of pulse trains from three different pulsars. CP 1919 shows a high amplitude correlation over about 50 pulses. CP 1133 shows each pulse to be uncorrelated with its neighbours.

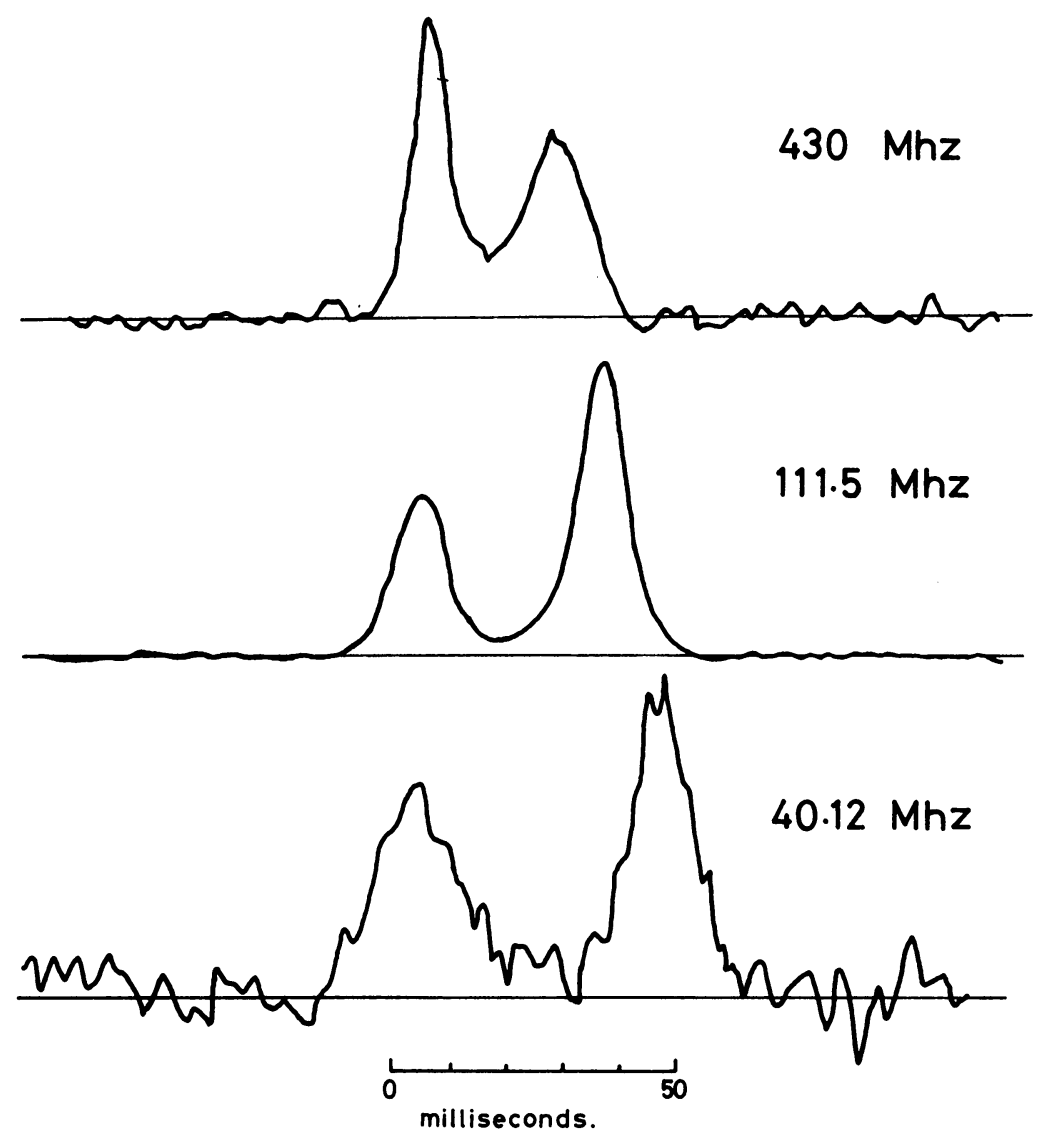

Fig. 7. The variation of the profile of CP 1133 with frequency (Craft and Comella).

CP 0328 has amplitude correlation over about three pulses followed by a negative correlation. In this case, therefore, clusters of strong pulses are usually associated with neighbouring regions of weak pulses.

The pulse energy distributions mostly show an exponential form. NP 0532 is apparently rather strange in this respect in that it appears to have at least two distinct distributions, each apparently associated with different parts of the pulse. 


\section{Sub-Pulse Structure}

The variations in pulse profile from one pulse to the next are considerable in most pulsars, and the structure may be very complicated.

A marked regularity in the pulses from CP 1919 and AP 2015 was noticed by Drake and Craft (1968) in which features appeared to persist from one pulse to the next, drifting slowly earlier through the mean profile. In CP 0328 the features may drift either earlier or later. Typically they take ten pulse periods to drift through the pulse.

This effect can only be clearly observed when several successive pulses are observable. In the case of the Crab which gives only single isolated strong pulses it has not been possible to identify any such drifting. In this respect it is with the majority of pulsars.

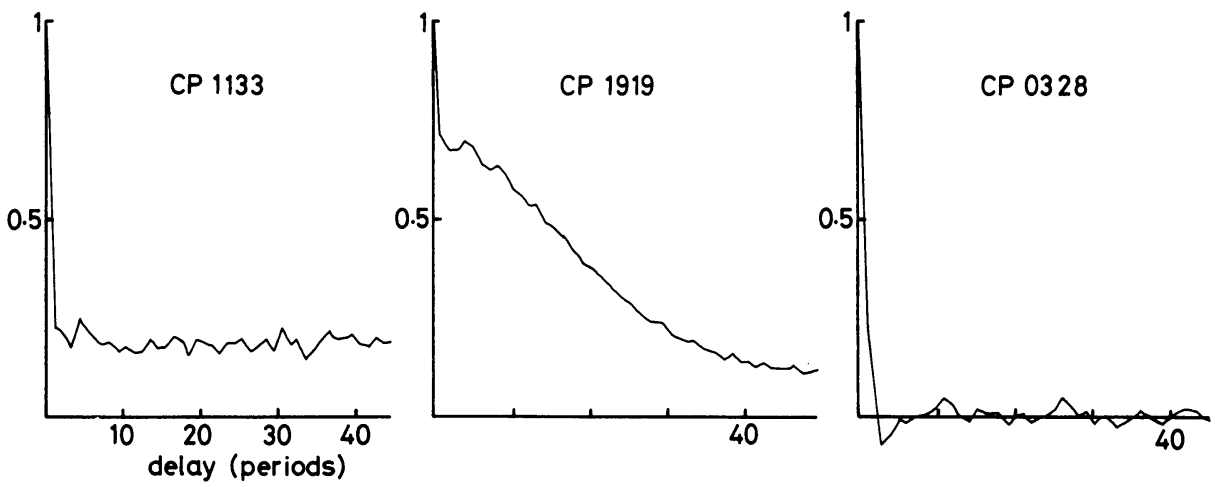

Fig. 8. The autocorrelation functions of the amplitudes of pulses from three pulsars.

\section{The Spectra of Pulsars}

There is much evidence that the radiation mechanism is broadband. With most pulsars, a large pulse on one frequency occurs as a large one on another frequency some octaves away. We heard yesterday that this was not strictly the case with NP 0532 .

Spectral measurements are complicated by interstellar scintillation but can be made by integration over large time or frequency intervals. The evidence is that for frequencies over $300 \mathrm{MHz}$ most pulsar intensities decrease as $f^{-2}$ or faster. There are a few notable exceptions which have much flatter spectra and are strong at high frequencies. JP 1933 and PSR 0833-45 are two such pulsars. CP 0328 has a peak in its spectrum between $400 \mathrm{MHz}$ and $1000 \mathrm{MHz}$. The Crab pulsar, NP 0532, has a spectral index of about -3.0 over the radio band which is larger than most other pulsars.

Over several months, the intensities of some pulsars change by considerable factors (Cole et al., 1970). As far as I know, there is no indication if this is due to a change in shape of the spectrum. 


\section{Interpulses}

Three pulsars are known to have interpulses - regions of impulsive emission roughly midway between the main pulses. CP 0950 and PSR 1929 have interpulses containing one or two percent of the power in the main pulse. The interpulse in NP 0532 is only slightly smaller than the main pulse, and in this respect is unlike any other pulsar.

\section{Conclusion}

This short review summarises most of the more obvious properties of pulsars. NP 0532 is unique in only a few respects. It has a remarkably short period, emission outside the radio band, strange amplitude variations and an abnormally large interpulse.

\section{References}

Cole, T. W., Hesse, H. K., and Page, C. G.: 1970, Nature 225, 712.

Craft, H. D. and Comella, J. M.: 1968, Nature 220, 676.

Drake, F. D. and Craft, H. D.: 1968, Nature 220, 231.

Hunt, G. C.: 1969, Nature 224, 1005.

Radhakrishnan, V. and Manchester, R. N.: 1969, Nature 222, 228.

Reichley, P. E. and Downs, G. S.: 1969, Nature 222, 229.

Reichley, P. E., Downs, G. S., and Morris, G. A.: 1970, Astrophys. J. 159, L35.

\section{Discussion}

C. Heiles: What is responsible for the differences between your relation for pulse width $v s$ pulse period and the one Dr. Drake showed to us yesterday, specifically in the cases of AP 1541 and NP 0532 ?

A. G. Lyne: The difference comes I think, from the method of measuring pulse width. I have used the area under the pulse divided by the peak intensity as a measure of effective pulse width for all pulsars even if they have two or more peaks.

J. Rankin: Although pulse broadening has now been observed in several other pulsars which is well described by an exponential $\left(e^{-x}, x \geqslant 0\right)$ broadening function, we wish to point out that this result is not perfectly general. Three observational properties of the pulsar, its low frequency pulse shapes, its spectral cut off at about $100 \mathrm{MHz}$, and its dispersion relation all indicate that a broadening function of the form $x e^{-x}$ is more appropriate than the simple exponential.

Arguments relative to the broadening based on the above three pulsar properties are necessarily a little indirect; however, recent observation of strong single pulses at $111.5 \mathrm{MHz}$ (with time bandwith product greater than 20) with good time resolution (about $3 \mathrm{~m} \mathrm{sec}$ ) allow observation of the broadening function direct. Such single pulses have widths of the order of $17 \mathrm{~m} \mathrm{sec}$ with the peak falling approximately $11 \mathrm{~m} \mathrm{sec}$ after the start of the pulses. Further, if the $f^{-2}$ component of the delay is extracted, it is found that the start of the strong pulse follows the intrinsic main pulse by about $2 \mathrm{~m}$ sec. Thus, although we cannot argue at this time that the strong pulse observation precisely support the $x e^{-x}$ broadening function hypothesis, we do argue that it is incompatible with an exponential broadening function.

F. G. Smith: Is it possible that the true pulse shape varies so much with frequency that part of your apparent smearing shape is really intrinsic? - Of course I realise that it must be possible - I ask is it reasonable? 
J. Sutton: In reply to Dr. Rankin's comment concerning shapes of individual pulses from NP 0532 at $111 \mathrm{MHz}$, our observations (as described in Paper 2.5, p. 97) are inconsistent with pulse shapes of the form $t e^{-a t}(t>0)$ but are better described by a shape $e^{-a t}(t>0)$ as consistent with electron scattering between the pulsar and us.

$M . M$. Komesaroff: The form of the scattering medium may determine the form of the scattering function. If it occupies a very much smaller depth than the distance between pulsar and observer I believe the scattering function will have an approximately exponential decay. 